\title{
Enhancing photovoltaic output power by 3-band spectrum-splitting \& concentration using a diffractive micro-optic
}

\author{
Nabil Mohammad, ${ }^{1}$ Peng Wang, ${ }^{1}$ Daniel J. Friedman, ${ }^{2}$ Rajesh Menon, ${ }^{1, *}$ \\ ${ }^{1}$ Department of Electrical \& Computer Engineering, University of Utah, Salt Lake City, UT 84112 \\ ${ }^{2}$ National Renewable Energy Laboratory, Golden, CO 80401 \\ *Corresponding author: rmenon@eng.utah.edu \\ Published XX Month XXXX
}

This document provides supplementary information to "Enhancing photovoltaic efficiency by 3-band spectrumsplitting \& concentration using a diffractive micro-optic", Optica volume, first page (year). The proposed microoptic was designed using a modified version of direct-binary-search algorithm and optimized using an optoelctronic model. Details of the design algorithm are presented in this document. Simulation results illustrating improvement in electrical performance of the photovoltaic cells, obtained using this algorithm, is discussed. Detailed information on fabrication process of the optic, i.e., from spin coating of photoresist to patterning by grayscale lithography is mentioned. Measurement of pixel heights using contact profilometer and corresponding error analysis is also included in the discussion. Finally, optical and electrical characterization process is presented in details. (C) 2014 Optical Society of America

http://dx.doi.org/10.1364/optica.99.099999.s1 [supplementary document doi]

To understand the design principle of the polychromat, let us consider the diffractive optical element (DOE) illustrated in Figure S1(a). The DOE is 1D, i.e., pixelated along the $\mathrm{X}$ direction with $(\mathrm{M}+1)$ pixels and uniform along the $\mathrm{Y}$ direction. Each pixel has a width of $\Delta \mathrm{x}$ and the height is multiple of a step height $\Delta \mathrm{h}$. The DOE's height profile is given by:

$$
h(\mathrm{x})=\sum_{m} \Delta h p_{m} \operatorname{rect}\left(\frac{x-m \Delta_{x}}{\Delta_{x}}\right)
$$

In Equation (S1), $\Delta h=H / N, \mathrm{H}$ is the maximum height, $\mathrm{N}$ is the number of levels; $p_{m} \in[0, N] \cap Z$; rect 0 is the rectangle function; $m \in[-$ $M / 2, M / 2]$ is the pixel index. The transmission function of such DOE can be expressed as $[1,2]$ :

$$
T(\mathrm{x} ; \lambda)=\mathrm{e}^{\mathrm{i} \varphi(\mathrm{x} ; \lambda)}=1+\sum_{m} \operatorname{rect}\left(\frac{x-m \Delta_{x}}{\Delta_{x}}\right)\left(\mathrm{e}^{\mathrm{i}(\lambda) \mathrm{p}_{\mathrm{m}}}-1\right)
$$

Here, $a(\lambda)=k \Delta h(n(\lambda)-1), n(\lambda)$ is the refractive index of the DOE material and $k=2 \pi / \lambda$ is the wave number. When a plane wave is normally incident upon the DOE, the diffracted field at the reconstruction plane for a particular wavelength $\lambda$ is given by the Fresnel transformation [1, 2]:

$$
U\left(\mathrm{x}^{\prime} ; \lambda\right)=\frac{e^{i k d}}{i \lambda d} \int g_{\text {illum }}(\mathrm{x} ; \lambda) \mathrm{T}(\mathrm{x} ; \lambda) \mathrm{e}^{\mathrm{i} \frac{k}{2 d}\left(\mathrm{x}^{\prime}-\mathrm{x}\right)^{2}} \mathrm{dx}
$$

In Equation (S3), $d$ is the distance between the DOE and the reconstruction plane (see Figure S1(b))and gillum $(x ; \lambda)$ is the power spectrum of the incident light.

In case of the polychromat, $M=5000, \Delta_{x}=3 u m, H=1.5 u m, N=64$, $\mathrm{d}=200 \mathrm{~mm}$. Minimum value of $\Delta_{\mathrm{x}}$ and maximum values of $\mathrm{H}$ and $\mathrm{N}$ are constrained by the fabrication process. A super-continuum source (NKT Photonics) was used as the input light spectrum. Power spectra of this source is illustrated in Figure S1(c). Shipley 1813 photoresist was chosen as the polychromat material. Woollman Ellipsometer was used to measure the dispersion data of the photoresist. This is shown in Figure S1(d). As mentioned in the main article, the height profile of the optic was optimized by maximizing the figure of merit (FOM), defined in Equation (1), by means of a modified version of the direct binary search algorithm [2]. In this algorithm, an initial height profile is chosen at the beginning. As the convergence of the solution is sensitive to initial condition, it is chosen carefully. Details on choosing the initial condition have been presented in [2]. Based on the initial condition, the diffracted field is calculated with the aid of Equation 
(S3). This is followed by calculating the short circuit current densities defined as [3]:

$$
\begin{aligned}
J_{s c, p o l y}^{c e l l, i} & =\iint \frac{q \lambda}{h c} I_{p o l y}(\mathrm{x}, \lambda) E Q E^{c e l l, i}(\lambda) d x d \lambda \\
J_{s c, r e f}^{c e l l, i} & =\iint \frac{q \lambda}{h c} I_{r e f}(\mathrm{x}, \lambda) E Q E^{c e l l, i}(\lambda) d x d \lambda
\end{aligned}
$$

In Equation (S4), $I_{p o l y}(X, \lambda)$ is the light intensity at point $X$ of the image plane for wavelength $\lambda$ in the case where the diffracted field is calculated at the presence of polychromat; $I_{r e f}(X, \lambda)$ in Equation (S5) is the reference intensity distribution, i.e., when the array of the solar cells are illuminated directly, i.e., the power spectrum of the source; cell, $i$ is ith solar cell, specifically cell1, cell2 and cell3 represent GaInP, GaAs and Si solar cells, respectively; $E Q E^{c e l l, i}$ is the external quantum efficiency of the ith solar cell; thus $J_{s c, r e f}$ and $J_{s c, p o l y}$ are the short circuit current densities when the solar cells are illuminated with and without the polychromat, respectively. From the short-circuit current densities, corresponding power densities are calculated as follows:

$$
\begin{gathered}
P_{\text {poly, }, i}=J_{s c, \text { poly }}^{\text {cell },} V_{o c}^{\text {cell, } i} F F^{\text {cell }, i} \\
P_{\text {ref }, i}=J_{s c, \text { ref }}^{c e l l, i} V_{o c}^{\text {cell, }, i} F F^{c e l l, i}
\end{gathered}
$$

In Equation (S6) and (S7), $V_{o c}$ and $F F$ stand for open circuit voltage and fill factor, respectively. To calculate the open circuit voltage, the I-V characteristics of each cell was measured first under direct illumination from the source. The measured open circuit voltage was then scaled to obtain the value for simulation using the following equations:

$$
V_{o c}^{c e l l, i}=\frac{n_{i} K T}{q} \ln \left(\frac{J_{s c, \text { ref }}^{c \text { cll, }}}{J_{s c, \text { meas }}^{c e l l, i}}\right)
$$

Ideally, $J_{s c, r e f}$ and $J_{s c \text { meas }}$ should be same. However, there is slight difference in the values of these two currents. One reason for the discrepancy can be attributed to the lower irradiance value of the light incident on the cells compared to the values used in the simulation. This is due to the fact that light is propagated over a long distance before illuminating the cells to ensure collimated input. Series and shunt resistance of the cells further lower the short circuit current density and open circuit voltage. Fill factor was also calculated from the measured I-V characteristics. Although spectrum splitting changes the open circuit voltage and fill factor slightly, this was neglected in the simulation and same value was used in both Equation (S6) and (S7) for both parameters.

Thus figure of merit is calculated for the initial height distribution utilizing Equation (S2)-(S8) and Equation (1). Once the initial FOM is calculated, the algorithm selects one pixel at a time and perturbs the initial height of that pixel by $\Delta \mathrm{h}$. Then the FOM is evaluated for the updated height. If the new FOM is higher than the original one, the perturbation is accepted otherwise the height of the pixel is returned to its original value. In this manner every pixel of the polychromat is perturbed and a loop is completed. This process continues until none of the perturbation of the pixels results in improved FOM. The process can also terminate when the improvement in the FOM is less than a defined tolerance value.

Using this algorithm an optimized design of polychromat was obtained. The resulting improvements in short circuit current densities and peak output power densities are illustrated in Figure S2.

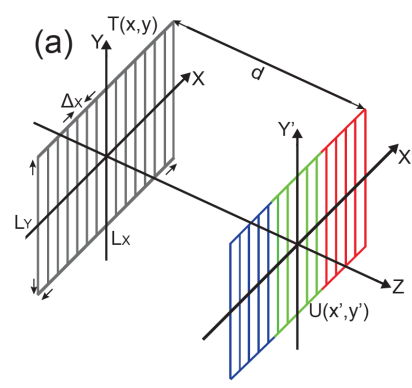

(b)
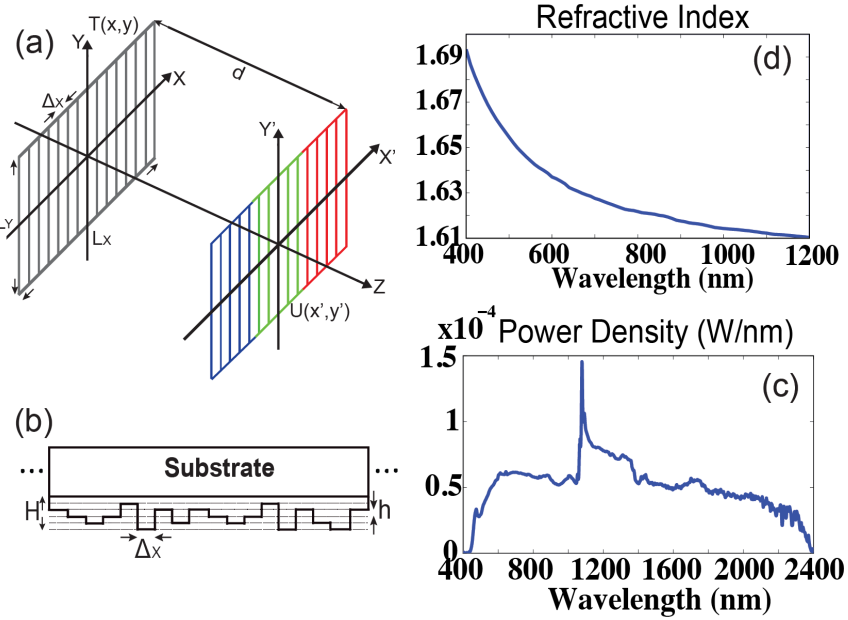

Fig. S1. Design principle of the diffractive optical element (polychromat). (a) Spectrum-splitting model in the Fresnel domain (gray grids for the micro-optic; blue, green and red for GaInP, GaAs and Si cells, respectively). (b) Schematic representation of the polychromat (cross-section in the X-Y plane). (c) Measured refractive index as a function of wavelength. (d) Power spectra of the supercontinuum source used in optical and electrical characterization
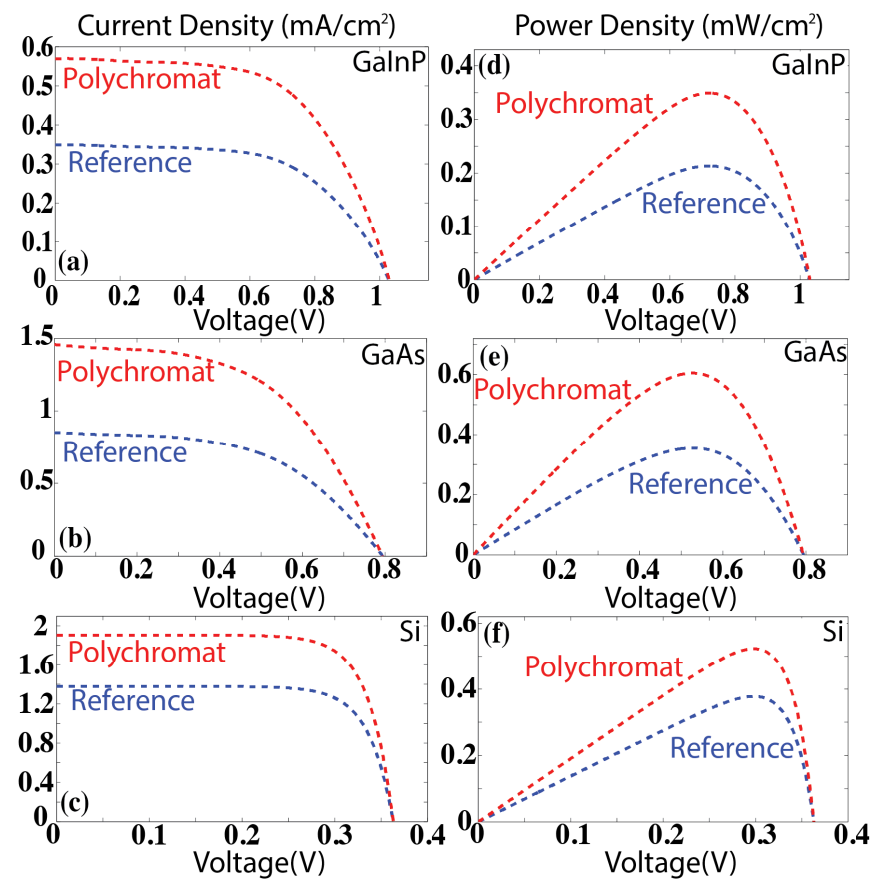

Fig. S2. Simulation results. Current density vs voltage for (a) GaInP (b) GaAs and (c) Si cells. Power density vs voltage for (d) GaInP (e) GaAs and (f) Si cells. Reference results are shown in blue, while spectrumsplit results are in red.

The polychromat was fabricated by patterning Shipley 1813 photoresist spin coated on a 3" square glass substrate. The glass substrate was RCA cleaned first. The photoresist was then spin coated on the glass substrate at a speed of $2000 \mathrm{rpm}$ for 60 seconds. This produced a film of thickness of about $1.6 \mu \mathrm{m}$. The sample was then baked in an oven at $110^{\circ} \mathrm{C}$ for 40 minutes. The photoresist was exposed by the MicroPG 101 pattern generator from Heidelberg Instruments. Before writing the polychromat design, calibration was done on a separate sample prepared in a same manner. Calibration results are shown in Figure S3(a). The design shown in Figure S3(b) was used for calibration. As the gray 

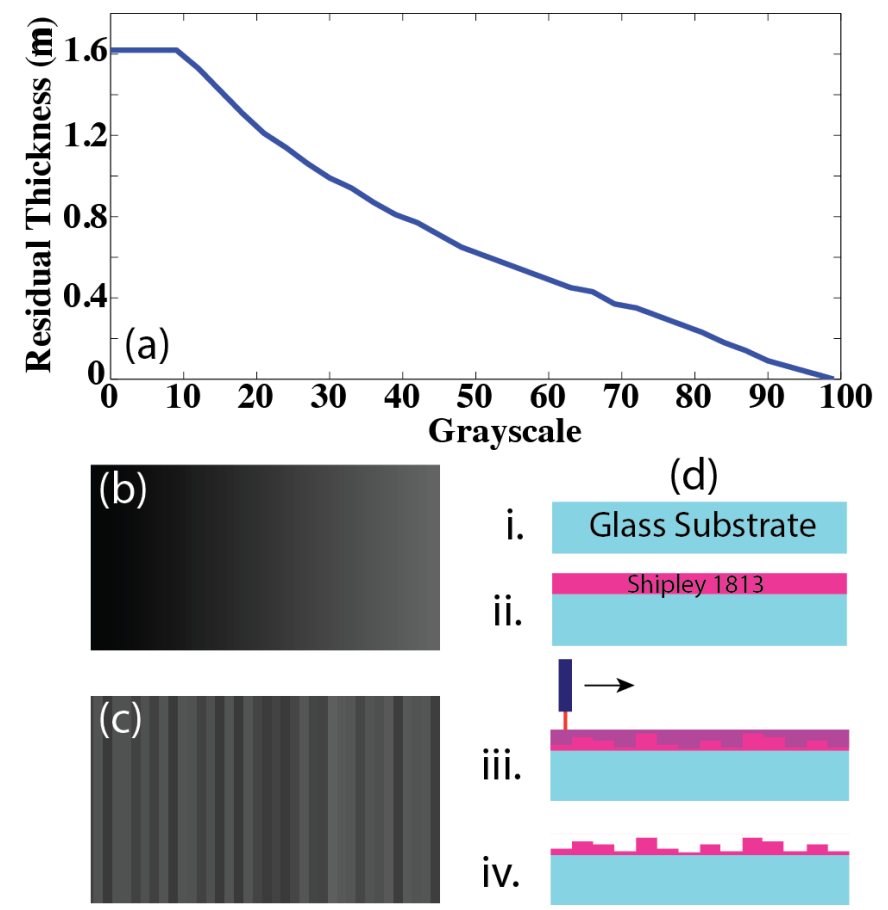

(d)

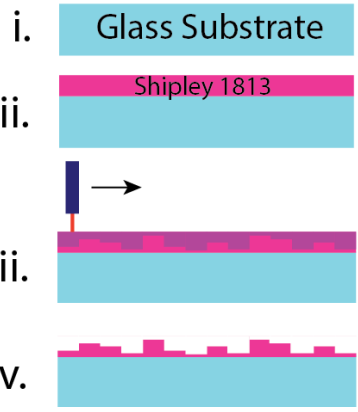

Fig. S3 Fabrication of the polychromat. (a) Calibration measurements: residual thickness of the photoresist as a function of grayscale. (b) Design file for calibration. (c) A small segment of the polychromat design file. (d) Fabrication process flow: (i) RCA cleaned glass substrate, (ii) spin coating of Shipley 1813, (iii) exposing the photoresist using MicroPG 101 (laser power is modulated according to the gray levels in the design) and (iv)multilevel structure following development.
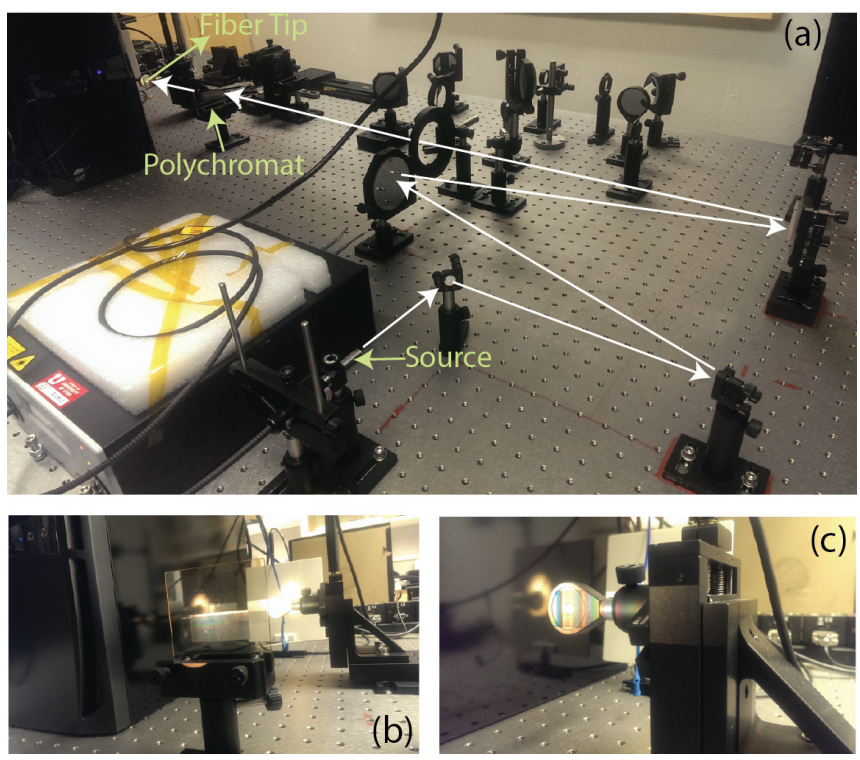

Fig. S4. Experimental setup for optical and electrical characterization. (a) Optical setup for guiding light from source to the polychromat (Light path is showed by white arrows). (b) Polychromat mounted on a holder. (c) Fiber tip mounted on a motorized step.

levels in the calibration design change from 0 to 99 , the laser power incident on the photoresist increases accordingly, resulting in thinner residual film. This calibration was used to translate the polychromat height distribution obtained from simulation to gray scale values in the mask design. The polychromat design (Figure S1(c)) was then written on the photoresist with same dose of the laser used for calibration. Finally, the sample was developed in 352 developer for 1 minute and a multilevel structure was left on the photoresist. The fabrication process flow is illustrated in Figure S3(d). To investigate the fabrication error, three segments, each consisting of 50 pixels, were chosen at three different locations along the X direction of the polychromat. Height of each pixel was measured using Tencor Profilometer. The error was calculated by subtracting the measured heights from the design heights. The mean of the error was $\sim 10 \mathrm{~nm}$ and the standard deviation was $\sim 141 \mathrm{~nm}$.

The polychromat was optically characterized by measuring its spatial-spectral intensity distribution at the image plane. The spatial-spectral intensity distribution is defined as [3]:

$$
S(\mathrm{x}, \lambda)=\frac{I_{p o l y}(\mathrm{x}, \lambda)-I_{\text {dark }}(\mathrm{x}, \lambda)}{I_{r e f}(\mathrm{x}, \lambda)-I_{\text {dark }}(\mathrm{x}, \lambda)}
$$

In Equation (S9), $I_{\text {poly }}(x, \lambda)$ and $I_{\text {ref }}(X, \lambda)$ represent the spatialspectral intensity distribution at the image plane when the polychromat is illuminated through the patterned and unpatterned photoresist respectively. This normalization is done due to the non-uniformity of the source spectrum. $I_{\text {dark }}(x, \lambda)$ is the measurement at dark and represents any background noise associated with the measurement process. For optical characterization the setup in Figure S4 was used. A 109mW supercontinuum source (SuperK COMPACT, NKT Photonics) was used for illumination. Light emanated from the source was guided to illuminate the polychromat design on the glass substrate which was mounted in such a way that the surface is perpendicular to the incident beam. A single mode fiber tip was placed at a distance of $200 \mathrm{~mm}$ and mounted on a motorized stage. The other end of the fiber was connected to Ocean Optics Jaz Spectrometer. The tip was scanned across $\mathrm{X}$ direction at the imaging plane with $10 \mu \mathrm{m}$ step. The measured data was normalized according to Equation (S9) to generate the spectral-spatial map shown in Figure 2(c). The map demonstrates the splitting of the spectrum into three bands. The spectrum splitting can also be observed by placing a white screen at the reconstruction plane.

For electrical characterization, GaInP, GaAs and Si solar cells were used to absorb the three diffracted bands. A glass support was used to hold these cells. The glass support was placed at the same distance $(200 \mathrm{~mm})$ at which the spatial-spectral map was measured. First the cells were visually aligned to the diffracted bands. Then the stage was moved laterally and I-V characteristics were measured for each cell at each position. The position that gave the highest peak power was chosen for final measurement. I$\mathrm{V}$ characteristic was measured using Keithley 2602A Source Meter. Figure 3 illustrates these measurements. The reference measurement was done in the same manner expect the light was guided through the unpatterned region of the photoresist on the substrate instead of the design area.

\section{References}

1. J. W. Goodman, Introduction to Fourier Optics (Roberts and Company Publishers, 2005)

2. G. Kim, J-A. Domínguez-Caballero, and R. Menon, "Design and analysis of multi-wavelength diffractive optics," Opt. Express 20, 2814-2823 (2012).

3. P. Wang, J-A. Dominguez-Caballero, D. Friedman and R. Menon, “A new class of multi-bandgap high efficiency photovoltaics enabled by broadband diffractive optics," Prog. Photovoltaics Res. Appl., DOI: 10.1002/pip.2516 (2014). 